ESAIM: Proceedings, Vol. 4, 1998, 73-81

Contrôle et Équations aux DÉrivées Partielles

http://www.emath.fr/proc/Vol.4/

\title{
REMARKS ON THE APPROXIMATE AND NULL CONTROLLABILITY OF SEMILINEAR PARABOLIC EQUATIONS
}

\author{
E. FERNÁNDEZ-CARA ${ }^{1}$ \\ Dpto. E.D.A.N., \\ University of Sevilla, \\ Aptdo. 1160, 41080 SEVILLA, SPAIN. \\ E-mail: cara@numer.us.es
}

Key Words : Semilinear Parabolic Equations, Null Controllabiblity, Approximate Controllability.

AMS Subject Qualification : 93C20, 93B05, 93B07, 35K20.

Dedicated to Jean-Pierre Puel on the occasion of his 50th birthday

\footnotetext{
${ }^{1}$ This work has been partially supported by D.G.I.C.Y.T. (Spain), Proyecto PB95-1242.

Article published by EDP Sciences and available at http://www.edpsciences.org/proc or http://dx.doi.org/10.1051/proc:1998021
} 


\begin{abstract}
In this contribution, we provide answers to the following two questions: 1) Which semilinear parabolic equations are approximately controllable (in the $L^{2}$ sense) ?

2) Which are null controllable?
\end{abstract}




\section{Introduction}

Assume a bounded and connected open set $\Omega \subset \mathbb{N}^{N}$ with regular boundary $\partial \Omega$, a nonempty open subset $\mathcal{O} \subset \Omega$ and a positive number $T$ are given. We will put $Q=\Omega \times(0, T), \Sigma=\partial \Omega \times(0, T), H=L^{2}(\Omega) ;|$.$| and (.,$.$) will stand for$ the usual norm and scalar product in $H$, respectively; $C$ will denote a generic constant.

We will be concerned with systems of the form

$$
\left\{\begin{array}{l}
\partial_{t} y-\Delta y+f(y)=1_{\mathcal{O}} v \text { in } \quad Q \\
y=0 \text { on } \Sigma \\
y(0)=y_{0}
\end{array}\right.
$$

where $y_{0} \in H$ and $v \in L^{2}(\mathcal{O} \times(0, T))$ (the control) are given. Here, $1_{\mathcal{O}}$ is the characteristic function of the set $\mathcal{O}$ and it is assumed that $f: \mathbb{N} \mapsto \mathbb{N}$ is locally Lipschitz-continuous.

For each $y_{0} \in H$ and each $v \in L^{2}(\mathcal{O} \times(0, T))$, let $S\left(y_{0} ; v\right)$ be the set of all solutions $y \in C^{0}(0, T ; H)$ to $(1)$; of course, $S\left(y_{0} ; v\right)$ can be the empty set. Let us also put

$$
R\left(y_{0} ; T\right)=\left\{y(T) ; y \in S\left(y_{0} ; v\right), v \in L^{2}(\mathcal{O} \times(0, T))\right\}
$$

the set of reachable states at time $T$. It will be said that (1) is approximately controllable (in $H$ at time $T$ ) if, for every $y_{0} \in H$, the set $R\left(y_{0} ; T\right.$ ) is dense in $H$. It will be said that null controllability holds for (1) if $R\left(y_{0} ; T\right) \ni 0$ for all $y_{0} \in H$.

In this contribution, our main goal is to provide answers to the following two questions:

1. Which are those functions $f$ such that the corresponding system (1) is approximately controllable?

2. Which are those $f$ such that (1) is null controllable ?

In general, when $f$ is superlinear at infinity, (1) is neither approximate nor null controllable. Indeed, recall that when $f(s)=-s \cdot(\log |s|)^{r}(r>1)$ blow-up phenomena may occur. Even when $f$ has "the good sign", approximate and null controllability may fail (see $[2],[3],[9]$ ). For instance, whenever

$$
C|s|^{r+1}-C \leq f(s) \cdot s \leq C|s|^{r+1}+C \quad(r>1)
$$

and $y_{0} \neq 0$, we can find a time $T_{0}>0$ such that $0 \notin R\left(y_{0} ; T\right)$ for all $T<T_{0}$.

On the other hand, when $f$ is sublinear at infinity, i.e.

$$
|f(s)| \leq C|s|+C
$$

the situation is completely different and (1) is both approximately and null controllable, with no restriction on $T$ or $\mathcal{O}$. This has been proved in [4] (resp. [3])

ESAIM: Proc., Vol. 4, 1998, 73-81 
in the case of approximate (resp. null) controllability. In both cases, the argument uses a fixed-point reformulation and the controllability properties of related linear problems. The key points are the unique continuation property in the case of approximate controllability and an observability inequality for null controllability. The assumption (2) leads to suitable estimates and allows to apply Schauder's theorem.

Hence, the natural question is: What happens if $|f(s)|$ grows faster than $|s|$ but slower than all $|s|^{r}(r>1)$ as $|s| \rightarrow \infty$ ?

We are also interested in questions of this kind for systems controlled on a portion of the boundary. More precisely, let $\gamma$ be a nonempty open subset of $\partial \Omega$ and let us put

$$
\Gamma=\partial \Omega \backslash \gamma .
$$

For each $h \in L^{2}(\Sigma)$, we consider the parabolic problem

$$
\left\{\begin{array}{l}
\partial_{t} y-\Delta y+f(y)=0 \quad \text { in } \quad Q, \\
y=1_{\gamma} h \text { on } \Sigma \\
y(0)=y_{0}
\end{array}\right.
$$

where $f$ is as before and $1_{\gamma}$ is the characteristic function of $\gamma$.

As before, we can introduce the notions of approximate and null controllability for (3). Thus, let $S\left(y_{0} ; h\right)$ be the set of all solutions to (3) which belong to $C^{0}(0, T ; H)$. Again, $S\left(y_{0} ; h\right)$ can be the empty set. Notice in particular that, for general data $y_{0} \in H$ and $h \in L^{2}(\Sigma)$, a "solution" $y$ to (3) does not necessarily satisfy $y(T) \in H$ (however, this is the case if $h \in L^{\infty}(\Sigma)$, see [4]; an example of this situation is given in [10], remark 9.3 in p. 217).

Let us put

$$
R\left(y_{0} ; T\right)=\left\{y(T) ; y \in S\left(y_{0} ; h\right), h \in L^{2}(\Sigma)\right\} .
$$

We will say that (3) is approximately controllable (resp. null controllable) at time $T$ if $R\left(y_{0} ; T\right)$ is dense in $H$ (resp. $R\left(y_{0} ; T\right) \ni 0$ ) for every $y_{0} \in H$. Then, under condition (2), system (3) is approximately and null controllable (cf. [3]; see also [1] for the case of Neumann boundary null controllability). It is also true that, for superlinear nonlinearities, (3) is neither approximately nor null controllable in general. Consequently, it is also natural to study the case in which $|f(s)|$ grows superlinearly but slower than all $|s|^{r}$.

\section{The null controllability result}

Concerning null controllability, let us recall the following result from [5]:

Theorem 2.1 Assume $f: \mathbb{N} \mapsto \mathbb{N}$ is locally Lipschitz-continuous, $f(0)=0$ and

$$
\lim _{|s| \rightarrow \infty} \frac{f(s)}{s \cdot \log |s|}=0 .
$$


Then (1) and (3) are null controllable.

Notice we are dealing with quasi-optimal assumptions: recall again that, for $f(s)=|s|^{r-1} s(r>1)$, systems (1) and (3) are not null controllable in general; also, recall that blow-up may occur for $f(s)=-s \cdot(\log |s|)^{r}$. The assumption $f(0)=0$ is completely reasonable. Otherwise, we would be trying to drive the system exactly to zero and, at the same time, a nonvanishing (nearly constant) forcing term would be acting on $\Omega$.

Sketch of the proof: We will only indicate the main ideas in the proof of this theorem in the case of the distributed control system (1). For simplicity, we will assume that $f$ is $C^{1}$ in a neighborhood of 0 and, also, that $y_{0} \in C^{1}(\bar{\Omega})$. Once theorem 2.1 is established under these assumptions, it is not difficult to deduce the same result in the general case; see [5] for the details.

Let us introduce the function $g$, with

$$
g(s)= \begin{cases}\frac{f(s)}{s} & \text { for } s \neq 0 \\ f^{\prime}(0) & \text { at } s=0\end{cases}
$$

In view of assumption (4), one has:

$$
\forall \delta>0 \quad \exists C_{\delta} \quad \text { such that } \quad|g(s)| \leq C_{\delta}+\delta|\log | s|| \quad \forall s \in \mathbb{N} .
$$

Let us set $Z=L^{\infty}(Q)$ and let us denote by $\|\cdot\|_{\infty}$ the usual norm in this space. We will need the following result:

Lemma 2.1 There exist a mapping $\Lambda_{0}: Z \mapsto Z$ and a positive constant $C_{0}=$ $C_{0}(\Omega, \mathcal{O}, T)$ such that:

1. For $a \in Z, y=\Lambda_{0}(a)$ means that $y$ solves, together with some $v \in L^{2}(\mathcal{O} \times$ $(0, T))$, the following null controllability problem (where the state equation is linear):

$$
\left\{\begin{array}{l}
\partial_{t} y-\Delta y+a y=v 1_{\mathcal{O}} \quad \text { in } \quad Q \\
y=0 \quad \text { on } \quad \Sigma \\
y(0)=y_{0}, \quad y(T)=0
\end{array}\right.
$$

2. The mapping $\Lambda_{0}: Z \mapsto Z$ is continuous and compact and satisfies

$$
\left\|\Lambda_{0}(a)\right\|_{\infty} \leq e^{C_{0}\left(1+\|a\|_{\infty}\right)} \quad \forall a \in Z
$$

A proof of this lemma can be found in [5]. There, for the construction of $\Lambda_{0}(a)$, some previous results from [7] and [8] were used. The continuity and compactness of $\Lambda_{0}$ were deduced from the regularity properties of the solutions to $(6)$, which use the fact that $y_{0} \in C^{1}(\bar{\Omega})$. Specifically, it was shown that $\Lambda_{0}$

ESAIM: Proc., Vol. 4, 1998, 73-81 
maps the bounded sets in $Z$ into bounded sets of $C^{0, \alpha, \alpha / 2}(\bar{Q})$. By definition, this is the Banach space formed by all functions $v \in C^{0}(\bar{Q})$ such that

$$
[v]_{\alpha, \alpha / 2}:=\sup _{\bar{Q}} \frac{\left|v(x, t)-v\left(x^{\prime}, t\right)\right|}{\left|x-x^{\prime}\right|^{\alpha}} \sup _{\bar{Q}} \frac{\left|v(x, t)-v\left(x, t^{\prime}\right)\right|}{\left|t-t^{\prime}\right|^{\alpha / 2}}<+\infty .
$$

For the obtention of (7), we need specific (global) Carleman inequalities for the solutions to the adjoint systems

$$
\left\{\begin{array}{l}
-\partial_{t} \varphi-\Delta \varphi+a \varphi=0 \quad \text { in } \quad Q \\
\varphi=0 \text { on } \Sigma
\end{array}\right.
$$

(with explicit estimates of the constants involved). Also, parabolic regularity results have to be used in the context of (6).

Let us put

$$
\Lambda(u)=\Lambda_{0}(g(u)) \quad \forall u \in Z .
$$

Obviously, $y=\Lambda(u)$ solves, together with some $v$, the null controllability problem

$$
\left\{\begin{array}{l}
\partial_{t} y-\Delta y+g(u) y=v 1_{\mathcal{O}} \quad \text { in } \quad Q \\
y=0 \quad \text { on } \quad \Sigma \\
y(0)=y_{0}, \quad y(T)=0
\end{array}\right.
$$

Consequently, in order to prove theorem 2.1 in this case, it suffices to check that Schauder's theorem can be applied to the fixed-point equation

$$
u=\Lambda(u), \quad u \in Z .
$$

But this is not difficult to see. Indeed, $\Lambda: Z \mapsto Z$ is again continuous and compact. Furthermore,

$$
\|\Lambda(u)\|_{\infty} \leq e^{C_{0}\left(1+\|g(u)\|_{\infty}\right)} \quad \forall u \in Z
$$

whence, using (5), it is easy to deduce that $\Lambda$ maps a ball of $Z$ into itself.

\section{The approximate controllability result}

In view of theorem 2.1, it is reasonable to expect approximate controllability for (1) and (3) under assumption (4). Again, this would provide a sharp criterion. The following result has been established in a joint work with E. Zuazua [6]:

Corollary 3.1 Assume $f: \mathbb{N} \mapsto \mathbb{N}$ is locally Lipschitz-continuous and (4) holds. Then (1) and (3) are approximately controllable.

Proof: Again, we only refer to the distributed control case; for further details, see [6]. Let us fix $y_{0}$ and $y_{1}$ in $H$ and $\varepsilon>0$. We have to find a couple $\{v, y\}$ satisfying (1) and the constrain $\left|y(T)-y_{1}\right| \leq \varepsilon$. For convenience, we take $y_{1}$ regular enough.

At this point, it is convenient to pay attention to the following remarks: 
1. Theorem 2.1 remains true, essentially with the same proof, when the nonlinear term is of the form $f(x, t, y)$, with $f: Q \times \mathbb{N} \mapsto \mathbb{N}$ being a Carathéodory function satisfying (for instance):

$$
f(x, t, 0) \equiv 0, \quad s \mapsto f(x, t, s) \quad \text { is locally Lipschitz }
$$

and

$$
\lim _{|s| \rightarrow \infty} \frac{f(x, t, s)}{s \cdot \log |s|}=0 \quad \text { uniformly in }(x, t) \in Q .
$$

Consequently, under assumption (4), one also has exact controllability to $L^{\infty}$ trajectories. In other words, if $w \in C^{0}(0, T ; H) \cap L^{\infty}(Q)$ satisfies

$$
\left\{\begin{array}{l}
\partial_{t} w-\Delta w+f(w)=0 \quad \text { in } \quad Q \\
w=0 \text { on } \Sigma
\end{array}\right.
$$

then there exists a couple $\{v, y\}$ satisfying (1) and $y(T)=w(T)$.

2. For each regular $y_{1}$, there exists $\delta>0$ such that the (unique) solution $Y$ to

$$
\left\{\begin{array}{l}
\partial_{t} Y-\Delta Y+f(Y)=0 \quad \text { in } \Omega \times(0, \delta) \\
Y=0 \text { on } \partial \Omega \times(0, \delta) \\
Y(0)=y_{1}
\end{array}\right.
$$

satisfies $Y \in C^{0}(0, \delta ; H) \cap L^{\infty}(\Omega \times(0, \delta))$ and $\left|Y(\delta)-y_{1}\right| \leq \varepsilon$. By definition, we set $Y_{\delta}=Y(\delta)$.

Now, we do the following:

- During the time interval $(0, T-\delta)$, we do nothing, i.e. we take $v=0$. This gives rise to a trajectory which starts from $y_{0}$ and describes a curve in $H$. The end-point of this curve is $y_{\delta}=y(T-\delta)$.

- By virtue of our first remark, there exists a couple $\{\hat{v}, \hat{y}\}$ satisfying

$$
\left\{\begin{array}{l}
\partial_{t} \hat{y}-\Delta \hat{y}+f(\hat{y})=\hat{v} 1_{\mathcal{O}} \quad \text { in } \quad \Omega \times(T-\delta, T) \\
\hat{y}=0 \text { on } \partial \Omega \times(T-\delta, T) \\
\hat{y}(T-\delta)=y_{\delta}, \quad \hat{y}(T)=Y_{\delta}
\end{array}\right.
$$

We take $v=\hat{v}$ for $t \in(T-\delta, T)$.

It is immediate that $v$ drives the system to a final state at a distance $\leq \varepsilon$ from $y_{1}$.

ESAIM: Proc., Vol. 4, 1998, 73-81 


\section{Some remarks}

In this final section, we present some comments and remarks on the previous controllability results.

First, let us indicate that several generalizations can be made. For example, there exists a positive constant $\varepsilon_{0}=\varepsilon_{0}(\Omega, \mathcal{O}, T)$ such that theorem 2.1 still holds with (4) or (8) replaced by the weaker assumption

$$
|f(x, t, s)| \leq \varepsilon_{0}|s| \cdot \log |s| \quad \text { for all }(x, t) \in Q \text { and large }|s| .
$$

One can also replace $-\Delta$ by a general linear elliptic operator $A$, with

$$
A y=-\partial_{i}\left(a_{i j}(x) \partial_{j} y\right)+\partial_{i}\left(b_{i}(x) y\right)+c(x) y,
$$

provided the coefficients are regular enough, etc.

As in the proof of corollary 3.1 , let us fix $y_{0}, y_{1}$ and $\varepsilon>0$. Since approximate controllability holds whenever (4) is satisfied, it is meaningful

$$
\begin{cases}\text { To minimize } & \frac{1}{2} \iint_{\mathcal{O} \times(0, T)}|v|^{2} d x d t \\ \text { subject to: } & \{v, y\} \text { solves }(1) \text { and }\left|y(T)-y_{1}\right| \leq \varepsilon .\end{cases}
$$

Problems of this kind are analyzed in [6].

Finally, observe that the arguments used in the proof of theorem 2.1 (and corollary 3.1) also serve, with appropriate changes, to investigate the controllability properties of some quasilinear parabolic systems. This will be reported in a forthcoming paper, written in collaboration with A. Dubova and M. GonzálezBurgos.

\section{References}

[1] D. Chae, O.Yu. Emanuilov, S.M. Kim: Exact controllability for semilinear parabolic equations with Neumann boundary conditions, to appear.

[2] O.Yu. Emanuilov: Thesis, Moscow 1991 (in russian); see also: Exact boundary controllability of the parabolic equation, Russian Math. Surveys, 48, No. 3 (1993), p. 211-212.

[3] O.Yu. Emanuilov: Boundary controllability of parabolic equations, Russian Acad. Sci. Sb. Math., 186, No. 6 (1995), p. 109-132.

[4] C. Fabre, J.P. Puel, E. Zuazua: Approximate controllability of the semilinear heat equation, Proc. Royal Soc. Edinbourgh, A (1995), 31-61.

[5] E. Fernández-Cara: Null controllability of the semilinear heat equation, ESAIM: Control, Optimisation and Calculus of Variations, April 1997, Vol. 2, pp. 87-103. 
[6] E. Fernández-Cara, E. Zuazua, in preparation.

[7] A.V. Fursikov, O.Yu. Emanuilov: On controllability of certain systems simulating a fluid flow, in Flow Control, IMA Vol. Math. Appl., 68, Ed. by M.D. Gunzburger, Springer-Verlag, New York (1995).

[8] A.V. Fursikov, O.Yu. Emanuilov: Local exact controllability for 2-D Navier-Stokes equations, Sbornik: Math., 187:9 1355-1390..

[9] J. Henry: Thèse, Université Paris VI, 1978.

[10] J.L. Lions: Contrôle optimal des systèmes gouvernés par des équations aux dérivées partielles, Dunod, Gauthiers-Villars, Paris 1968. 